\title{
"Appuhamy" - The Fully Automatic Rubber Tapping Machine
}

\author{
Y.A.I. Yatawara, W.H.C. Brito, M.S.S. Perera and D.N. Balasuriya
}

\begin{abstract}
Being a major export good from the colonial times, rubber still can greatly contribute to the national economy. However, lack of labour for rubber tapping has been a major factor contributing to the downfall of the Sri Lankan rubber industry. As a solution to the shortage of labour for rubber tapping we present the design of a fully automated rubber tapping machine which is capable of moving from tree to tree in a rubber plantation and tapping each tree automatically. Capability to automatically detect the tapping path, nodules, tree condition and many other important parameters makes the proposed rubber tapper much more superior over all existing rubber tapping machines and techniques.
\end{abstract}

Keywords: Rubber tapping, Fully automatic

\section{Introduction}

Rubber is a substance which possess special properties such as elasticity, high friction, softness, durability and very high electrical resistivity, hence is employed in an array of products used in our day to day life. From surgical gloves to automobile parts and from toys to electrical insulators, rubber is a key element in the production of nearly 50,000 products we use on a daily basis. To cater for this multi-fold requirement, today's world is producing approximately 26 million metric tons of rubber annually, out of which only 12 million is natural rubber [1]. To cater for the excess demand, 14 million tons of various synthetic rubber forms are also produced. Introduced to Sri Lanka by the Colonial English rulers, natural rubber has been a major export good of Sri Lanka even after the independence. Later, under the import substitution economic policies during 1972-1977, rubber became the key ingredient in many local industries such as Kelani Tyres and Richard Peris Rubber products. Even under the open economic policies during 1980 's, rubber retained its position as a major source of export income to the Island [2].

At the same time, many of the Asian countries such as India and Malaysia have steered themselves to be the world's largest natural rubber producers. Meanwhile, the technically advanced western world has focused their attention on the production of synthetic rubber which has overtaken the role of natural rubber over the last few decades. Due to its high - tech driven low cost mass production capability compared to the highly labour intensive small scale production of natural rubber, synthetic rubber is preferred wherever possible. Labour intensiveness in the tapping process for the natural rubber and also the introduction of synthetic rubber has paved the way to the downfall of the natural rubber industry in Sri Lanka. Furthermore, the export oriented industries like garments have further absorbed the labour force; hence rubber industry has become a dying industry in Sri Lanka.

Even though the synthetic rubber is a good substitution for many of the natural rubber based products, there are some products which still appreciate the unique properties of natural rubber. This requirement has paved way to a considerable market segment left for the developing nations with agriculture based economics to compete in. On the other hand, with a low level technological background, Sri Lanka is unable to compete with the synthetic rubber producers, but would be able to compete in the natural rubber market under sufficient technological involvement.

\footnotetext{
Eng. Y.A.I. Yatawara, AMIE(SL), B.TechEng.Hons(OUSL)

Engineer, Nokia Corporation Lanka (Pvt)Ltd, Comombo 01. Email:anushaindrajith@gmail.com

ORCID ID: https://orcid.org/0000-0002-8112-8801

Eng. W.H.C. Brito, AMIE(SL), B.TechEng. Hons(OUSL)

RED Engineer, Novena Tech (Pvt)Ltd, Dutugemunu St, Kohuwala.Email:britohima@gmail.com

ORCID ID: https://orcid.org/0000-0002-5017-424X

Eng. M.S.S. Perera, AMIE(SL), B.TechEng.Hons(OUSL) Lecturer, Department of Electrical and Computer

Engineering, The Open University of Sri Lanka, Nawala, Nugegoda. Email:sisaara.aera@gmail.com

ORCID ID: https://orcid.org/0000-0003-3747-3840

Eng. D.N. Balasuriya, CEng, $\operatorname{MIE}(S L), B S c$

Eng.Hons(Moratuwa), MSc (Manitoba),

Senior Lecturer, Department of Electrical and Computer

Engineering, The Open University of Sri Lanka, Nawala,

Nugegoda.Email:dnbal@ou.ac.lk

ORCID ID: https://orcid.org/0000-0002-0073-7091
} 
Thus, if a solution can be provided to the shortage of labour and if the rubber industry can be rehabilitated, there would be a considerable market and income for Sri Lankan natural rubber.

As a solution we present the design of a fully automated rubber tapping machine which can automate the process of rubber tapping reducing the labour requirement to a minimum. The rest of the paper is organized as follows. Section 2 discusses the currently available semiautomated rubber tapping systems around the world. In Section 3 we present the proposed fully automated rubber tapping machine design. In Section 4, we further elaborate on the functionality of the proposed rubber tapping machine followed by the prototype implementation details and the testing results. Finally, Section 5 concludes the paper highlighting several future research and development avenues.

\section{Automatic Rubber Tapping Systems}

During a fully manual rubber tapping process with the conventional rubber tapping knife, a tapper makes an incision in the bark of the rubber tree which causes the slow flow of the milky fluid "latex". This procedure is known as tapping. Latex is allowed to be collected in a container which is then collected and taken for processing. An individual tapper needs to visit each and every tree and it takes approximately five minutes to clean and tap a single tree and a further visit to each and every tree is also needed to collect the latex. With this labour intensive nature, an individual tapper may manage to tap only a few acres of rubber plantation per day.

To overcome this issue, several semi-automated rubber tapping machines have been proposed. The invention of the semi-automated rubber tapper at Palai College of Engineering Technology, India [3], which is based on a linear cutter and a manual fixing mechanism, is one such attempt. A tapping machine is to be fixed to each and every tree which carries out the movement of the cutting blade across a preprogrammed path. One of the major drawbacks of this machine is the high torque generated with the linear cutting which is not possible to be applied with a small motor. Moreover, this cutter is only developed at an experimental level and with the clogging properties of latex, the practical deployment of this cutter is doubtful. On the other hand, the need of a separate machine for each and every tree poses a cost disadvantage. Other considerable contribution in this area is from the semiautomated rubber tapping machine developed at the Velammal College of Engineering Technology, India [4].The key concept behind this machine is the introduction of the rotational cutter to produce a low torque cut. A worker needs to carry the machine from tree to tree to perform a cut but takes a lesser time to tap than in a manual tapping. Even then, this cutter too needs a considerable human involvement as it needs to be taken from tree to tree, very similar to the conventional manual rubber tapping process.

Therefore, in this research, we propose a fully automated latex tapping machine which neither needs to be carried from tree to tree manually nor needs to be programmed with the tapping path on a tree by tree basis.

\section{Design Methodology}

The proposed fully automated rubber tapping machine consists of several important sub units as depicted in Figure 1.

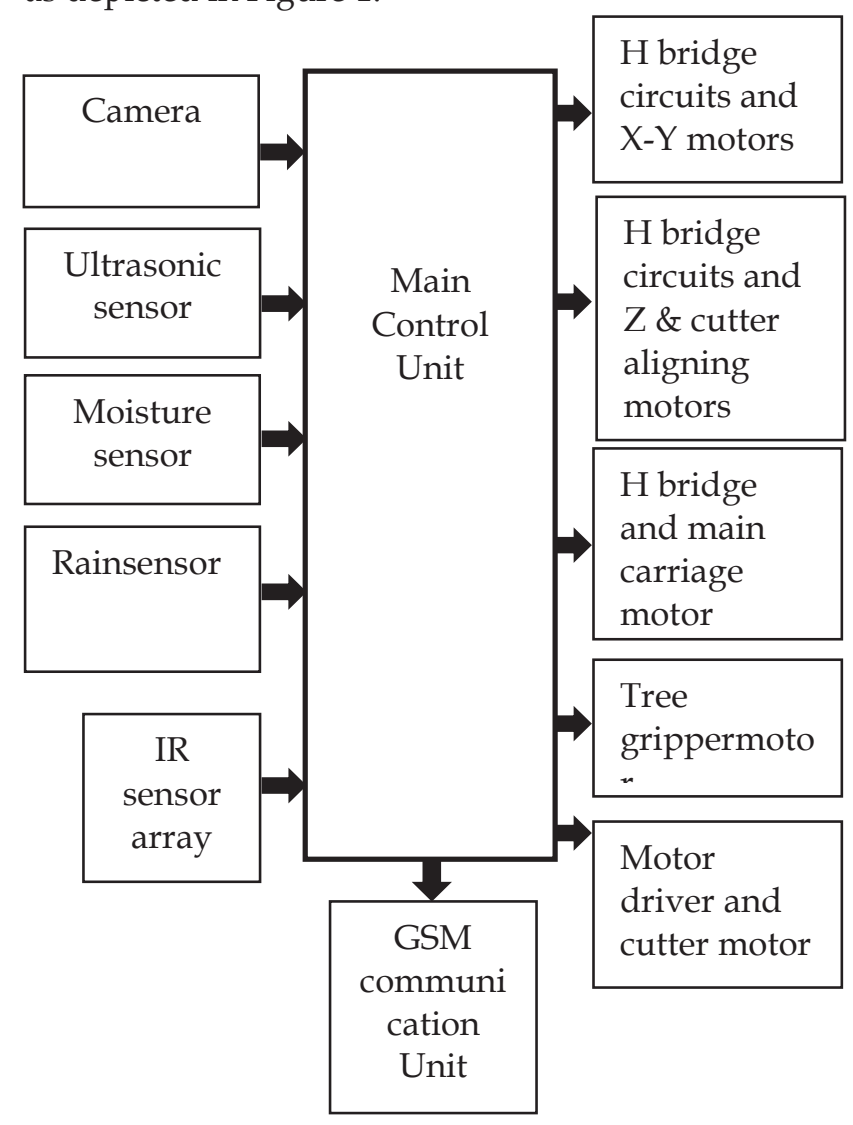

Figure 1 - System block diagram

The intelligent tapper head is one of the main components in this system which includes all the sensors and the camera and is capable of capturing the images of the rubber tapping path and determining the trajectory of the cut. 
It also detects the distance to the rubber tree and selects the depth of the cut. Moreover, the head carriage is a mechatronics arrangement which moves the tapping head along the tapping trajectory. At the same time, the main carriage is an arrangement to carry the rubber tapping machine from tree to tree during the tapping process. Note that all these units are controlled by a single controller unit.

\subsection{Intelligent Tapper Head}

The tapping process of a rubber tree is carried out along an inclined circular path along the rubber tree surface. Every day, a slim line of bark is removed along the cut together with the clogged latex, known as ottapalu, resulting from the previous cut. The intelligent tapping head mainly focuses on the identification of the tapping path of each and every rubber tree. This action is vital in the process of designing a fully automated rubber tapper as the automatic path detection enables the tapper travelling from tree to tree to identify the tapping trajectory without pre-programming.

The path identification mechanism is to be based on image processing. Several photographs around the tree are captured under constant lighting conditions, and the photographs are overlapped first to form a single image. Then the resulting image is converted to black and white via binary thresholding [5] and then inverted. The resultant black spaces along the tapping path are interpolated for a continuous trajectory and the resulting trajectory coordinates are recorded as a two dimensional data array as shown in Figure 2. The recoded trajectory is utilized for moving the cutter head during the tapping stage.

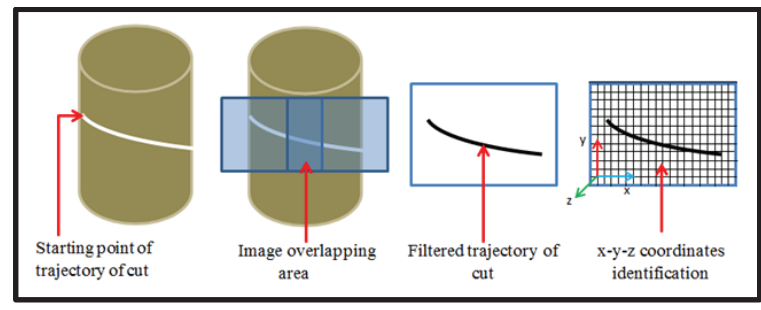

Figure 2 - Tapping tragectory identification

\subsection{Rotary Cutter and the Cutter Head Moving Mechanism}

One of the most innovative features of this system is the inclusion of a rotary cutter in view of a reduced torque. For efficient cutting, a slit with a slope to the horizontal on half a circumference of the particular rubber tree needs to be cut. Here, a fixed cutter consisting of four blades, was designed to allow the easy removal of bark. This cutter can be used to meet the industrial requirements and specifications in rubber tapping to maintain a 10 degree slope across the depth of the cut with the use of a tapered rotary cutter body. The cutter head can also be inclined accordingly using a separate gear wheel fixed to the fixture. This allows the tool to be always kept in an approximate perpendicular orientation to the tree surface.

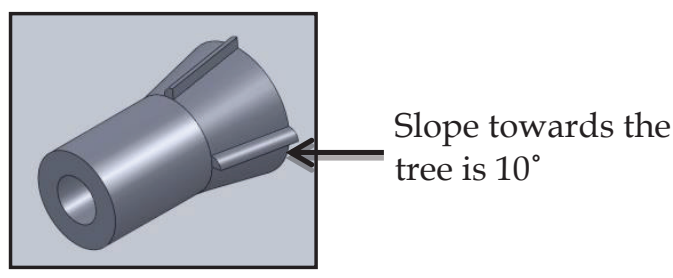

Figure 3 - Rotary cutter head

Moreover, it is highly recommended for the tapper to remove the bark of the tree completely to capture the total number of latex vessels, while not harming the inner tree which is known as the Cambium, as shown in Figure 4.

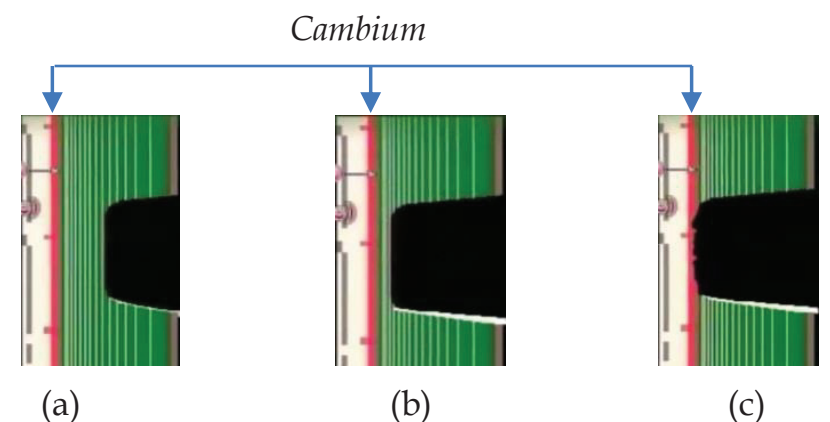

Figure 4 - The tapping cut (a) Under cut

(b) Optimal cut (c) Over cut

To cater for this requirement, in addition to the blades, the cutting head consists of a protruding $1.5 \mathrm{~mm}$ diameter semi global dotwhich prevents the cutter tool moving too far into the tree (Figure 5).

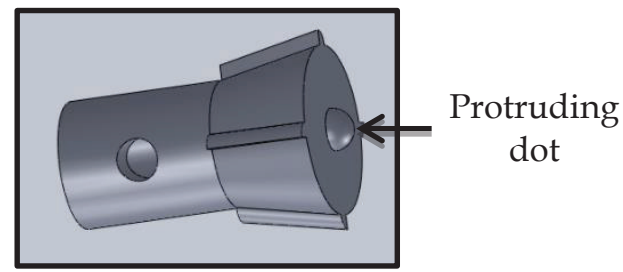

Figure 5 - Protruding dot arrangement

Another major issue that an automated tapping head faces is the unevenness in the depth to the tree surface from the two dimensional plane where the machine chassis is located. Thus, to cater for this issue, an inward and outward moving mechanism for the cutter head is 
proposed. This mechanism is aided by a depth detection mechanism implemented using an infrared (IR) sensor and an ultrasonic sensor fixed to a panel at the head [6].With the detected depth to the tree, the cutter will move in and out with respect to the tree (we denote this as $\mathrm{Z}$ movement). Even in the presence of nodules on the tree surface the above mechanism performs a precision cut without the need of any further hardware.

Meanwhile, it is of high importance to maintain the cutter approximately perpendicular to the tree surface throughout the cutting path. Note that the tapping head moves in an $X-Y$ plane while the cutter is capable of moving in the $\mathrm{Z}$ direction. Thus, we incorporate another degree of freedom for the cutter such that the cutter angle can be varied with the help of another motor and a gear system, within a range $\left[-90^{\circ}\right.$ to $\left.+90^{\circ}\right]$.

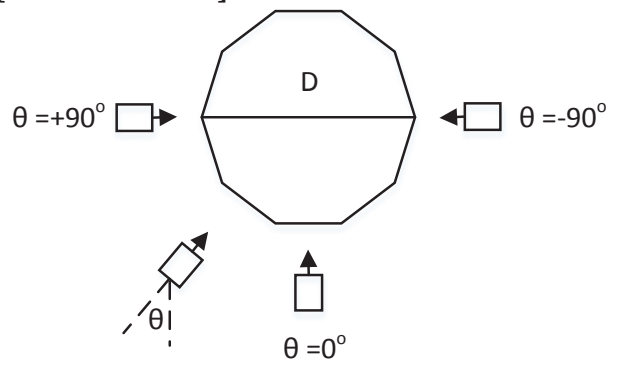

\section{Figure 6 - Proposed cutter angle aligning arrangement}

Cutter's angle to the $\mathrm{Z}$ direction given by $\theta$ is calculated and varied as, $\theta=\frac{180^{\circ} \times \delta x}{D}$, where $\delta x$ and $D$ are the horizontal displacement of the cutter head from the center of the $X$ direction trajectory and the total length of the $X$ direction trajectory, respectively (Figure 6). This technique enables the cutter to be maintained approximately perpendicular to the tree surface throughout the horizontal movement of the cutter head.

Design of the automated rubber tapper also involves the addition of a rotating tangler arm mechanism in front of the cutter to collect the clogged latex from a previous tapping. Furthermore, tree's surface moisture level sensor and a rain sensor are also included which are vital to avoid excess water in the clogged latex.

\subsection{Tapping Head Carriage}

Tapping head is fixed in a movable carriage which moves in a plane and in an inclined nonlinear path following the trajectory coordinates captured at the start. This special motion which we denote as $\mathrm{X}-\mathrm{Y}$ movement is achieved by appropriately varying the horizontal and vertical coordinates of the tapper head according to recorded two dimensional data array. Two motors fixed to two screwed shafts (controlled via H-bridge circuits [7]) produce the necessary horizontal and vertical motions (Figure 7). Furthermore, in order to match the speed requirements, a gearwheel arrangement is employed in this design.

Note that the design of a main carriage which performs an $X-Y$ movement in a plane rather than rotating around the tree is solely for the purpose of facilitating an easy tree to tree movement along the guide wires.

\subsection{Main Carriage}

The rubber tapping machine requires to be moving from tree to tree (Figure 8). Thus, a main carriage as in Figure 9 is employed to hold the rubber tapper, and the carriage moves along a pair of guide wires laid along the line of trees. At each tree, two steel arms connected to the rubber tree hold the steel cable. The carriage moves along these wires using a pulley mechanism as shown in Figure 9. Extra grooves on the pulleys, high tension in the steel wires and the shorter inter-tree distance below 5 meters ensure that the carriage does not get derailed while moving from tree to tree.

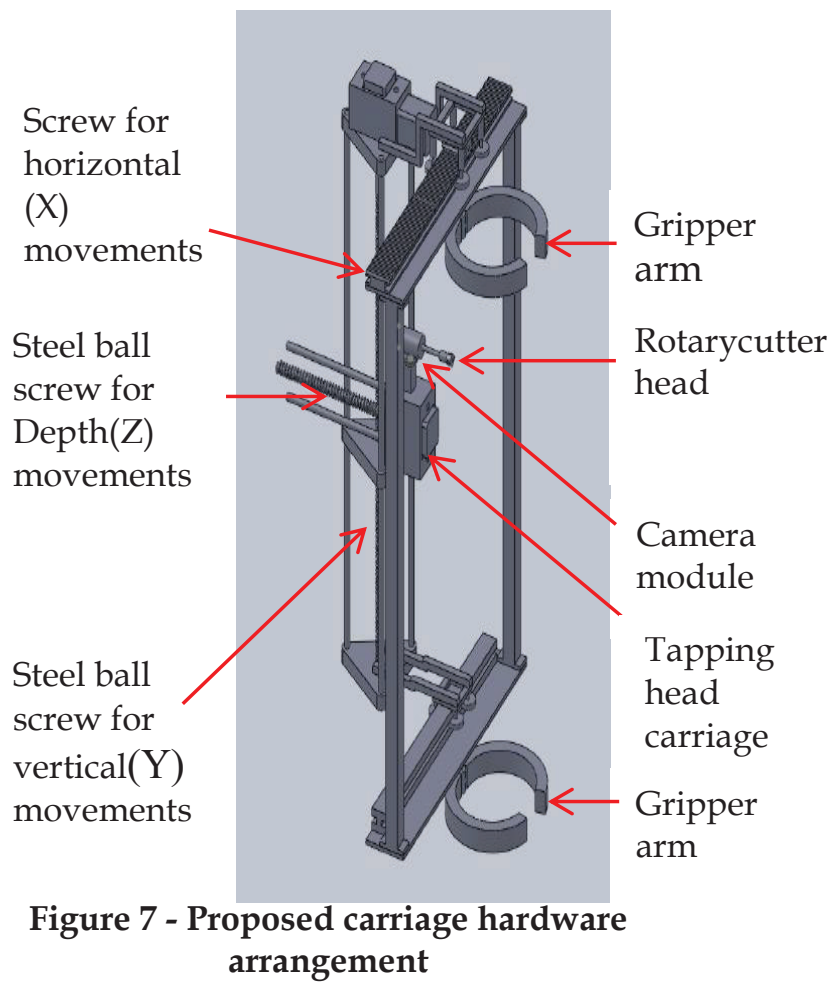

At a tree, two expandable gripper arms are operated to clamp the tapper to the tree. After the tapping process of the same tree is completed, these arms de-clamp from the tree 
so that the tapper can travel along the steel guide wire to the next tree.

The movement from tree to tree is achieved using an H-bridge controlled motor [7] fixed with a rubber belt; pushing against the steel guide wire forcing the carriage to move with respect to the wire due to friction.

Moreover, an automatic tree presence detection mechanism is implemented using an ultrasonic sensor fixed to the carriage facing the tree side of the line. Automatic tree presence detection and clamping control is to be controlled via the same control unit as employed for the tapper control. Furthermore, the GSM communication unit is used to notify a predefined mobile user when the carriage reaches the end of the line, thus has finished the tapping process.

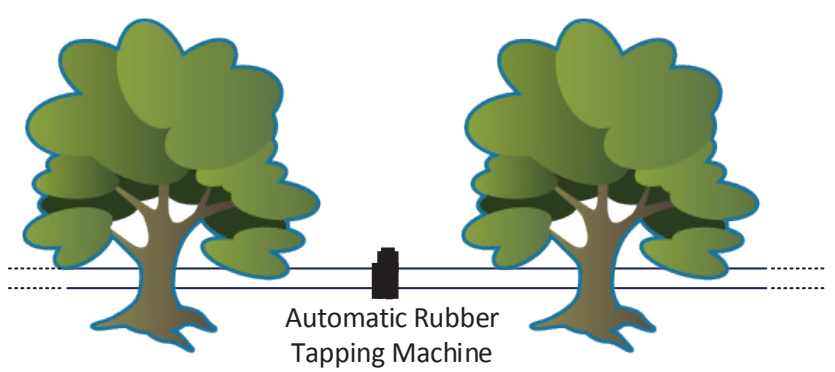

Figure 8 - Proposed fully automatic main carriage

\section{Operation and the Prototype Implementation}

The three sub systems are to be integrated together to perform a complete system which is capable of moving from rubber tree to another, identify the tapping trajectory, remove clogged latex and clean the tree, perform a cut and then move to the next tree.

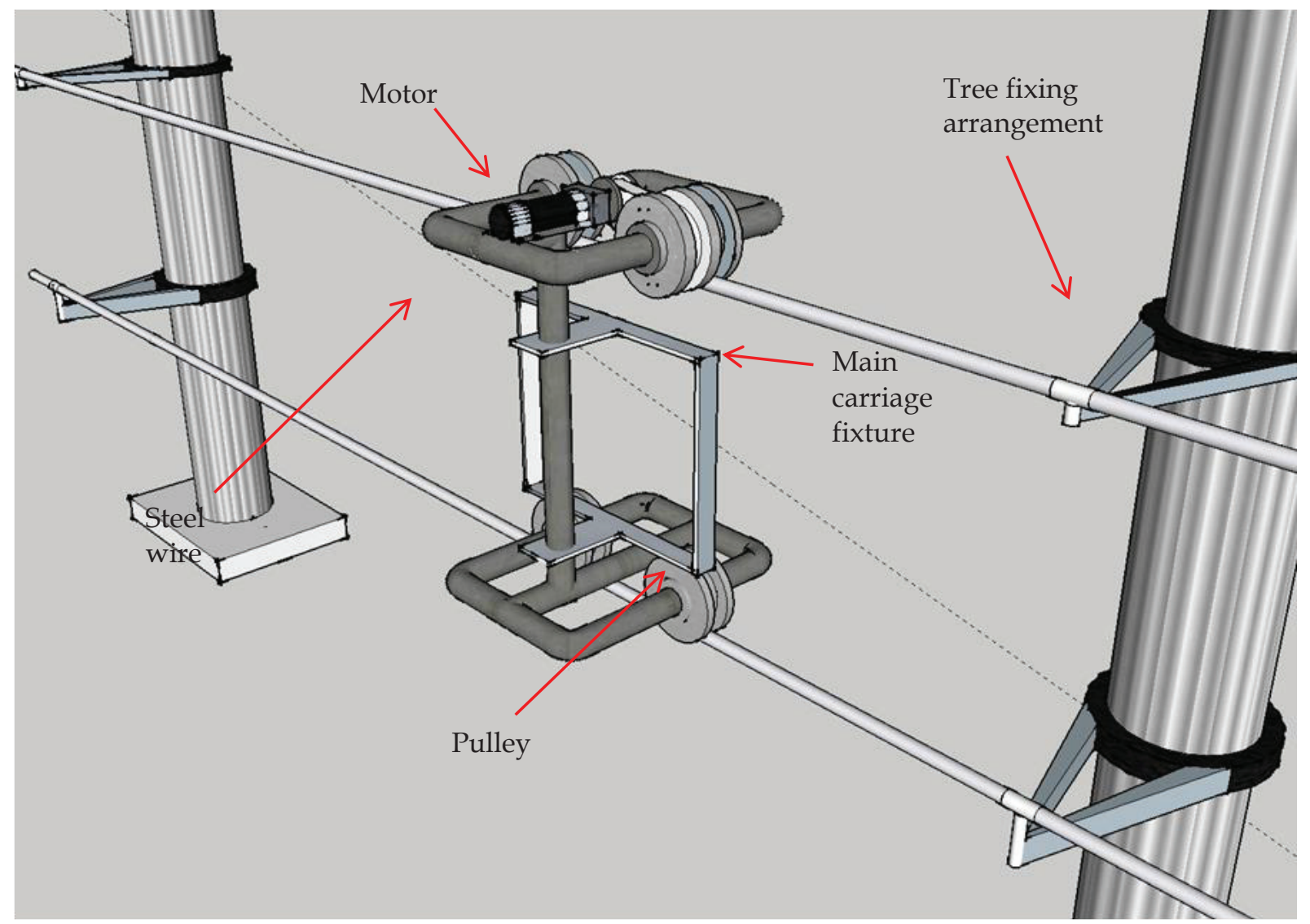

Figure 9 - Main carriage movement mechanism 


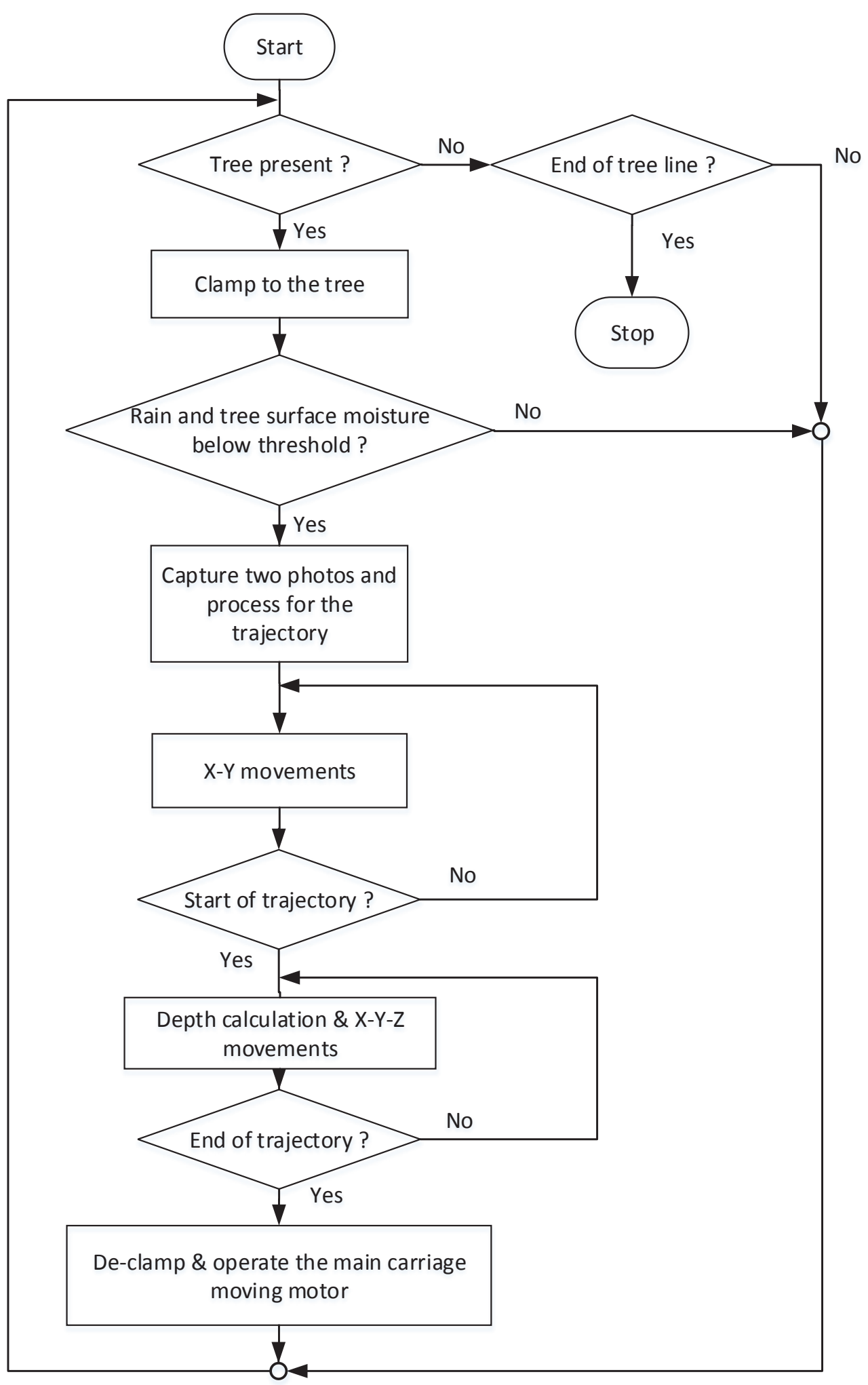

Figure 10 - Operational flow chart

The overall operation can be depicted as in Figure 10. In this design, the tapper head is operated by a $12 \mathrm{~V}, 3 \mathrm{~A}$ DC motor while the main carriage, head carriage and the tree to tree moving mechanism are operated using three $24 \mathrm{~V}, 3$ A stepper motors. All motors are driven using H-bridges and L298/DMD420 motor controller boards. Another $12 \mathrm{~V}$ DC motor is employed for providing the necessary torque for the rotational cutter. The system is operated with a $24 \mathrm{~V}, 600 \mathrm{Ahr}$ rechargeable battery pack.

Even though in young rubber plantations where the ground level receives sufficient sunlight to energize a solar cell generator, in mature plantations ground level gets very 
limited sunlight. Thus, the automated rubber tapping machine is designed to operate for several hours solely on the battery power. Furthermore, TCRT5000 IR sensor arrays and SRF02 ultrasonic range finders are employed in the machine for detections. Other key component in this machine is the camera, for which a high resolution 'Mind vision mvub500c'camera is selected together with automatic lighting control.

The controlling unit of the tapping machine is responsible for many functions including image processing for trajectory identification, controlling the horizontal, vertical and depth movements by driving the appropriate motors, and also identification of the presence of a rubber tree with an infrared proximity detector. Therefore, a Raspberry-pi minicomputer board is used in this controlling.

A prototype was implemented having all aforementioned units, but excluding the main carriage's moving mechanism, which weighed $24.2 \mathrm{~kg}$ including the battery (Figure 11).

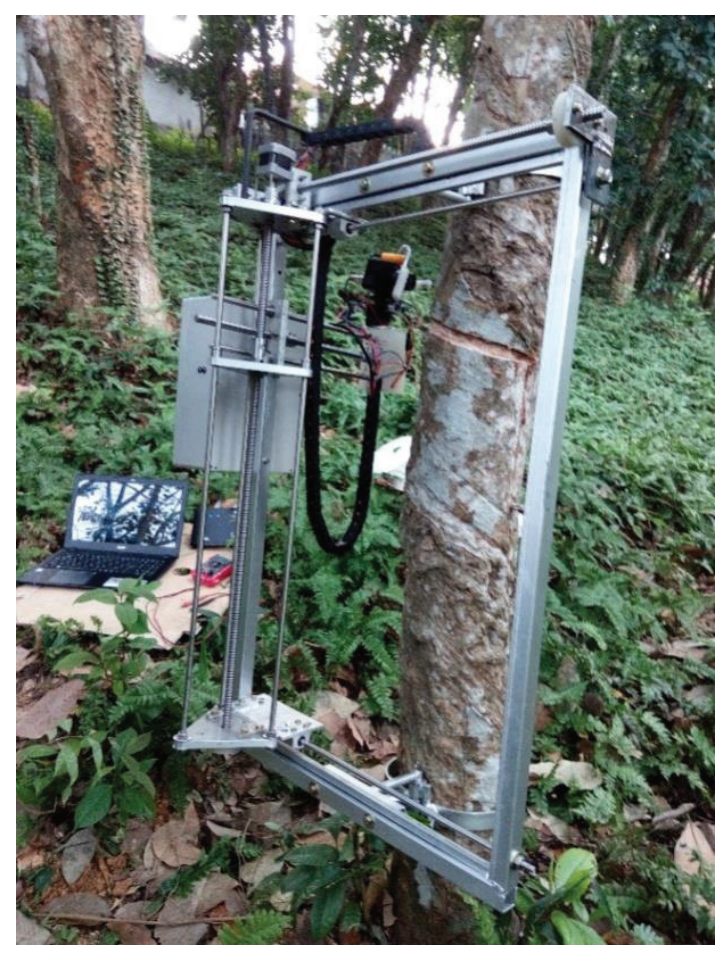

Figure 11 - Prototype implementation excluding main carriage movement mechanism

A sample run with the implemented prototype demonstrated an average tapping time of 48 seconds for one tree which is very much superior to the time spent by most of the existing tapping mechanisms.

\section{Conclusion}

The proposed automated rubber tapping system will be of immense value to the Sri Lankan rubber plantation sector as it avoids most of the major problems associated with the tapping process in an efficient way. A prototype implementation demonstrated its viability for a mass scale production at a fair cost.

However, there are several weaknesses in the proposed system which should be addressed in future improvements. One such weakness is the fact that the main carriage travels along a pair of guide wires. A proper mechanism to avoid the effect of wind to this movement is desired. At the same time, the $X-Y$ movements need to be slowed down in the presence of nodules, which is another issue to be addressed in future designs.

\section{Acknowledgement}

Authors wish to acknowledge the support extended by Eng. Rajeeva Perera in preparing the 3D drawings.

\section{References}

1. International Rubber Study Group, "Current Developments in the Rubber Industry", 2016.

2. The Central Bank of Sri Lanka, "Sri Lanka SocioEconomic Data", Vol XXXIII, 2010.

3. Joseph, P. V. "Automatic Rubber Tree Tapping Machine", Indian patent No. PCT/IN2011/000509/2012.

4. Malarmannan, G. R., Venkathesh, M., Richards, S. E. and Rajesh Kanna, P., "Semi Automated Rubber Tapping Machine", Indian patent No.3734/CHE/2012.

5. Sezgin, M. and Sankur, B. "Survey over Image Thresholding Techniques and Quantitative Performance Evaluation", Journal on Electronic Imaging,Vol.13,Issue 1, 2004, pp 146-165.

6. Srivasthava, A. K., Verma, A. and Singh, S. P., "Distance Measurement of an Object or An Obstacle by Ultrasound Sensors using P89C51RD2", International Journal of Computer Theory and Engineering,Vol.2, No.1,2010, pp 6468.

7. Zhu, Z., Kim, J. and Abbassian, K. "H-Bridge Drive Circuit for Step Motor Control", US patent No. US20110241597A1. 